\title{
Reabsorción radicular de incisivos laterales superiores en relación con la erupción ectópica de caninos. Presentación de dos casos
}

\section{Rodríguez Romero FJ*, Rodríguez Sánchez M**, Rodríguez Rodríguez B***}

\section{RESUMEN}

La impactación del canino superior permanente es un problema clínico que puede provocar una serie de secuelas que van desde la perdida de espacio en el arco para su correcta erupción, hasta la reabsorción radicular de dientes vecinos. El presente articulo describe dos casos de pacientes con ausencia clínica de caninos superiores, produciendo reabsorción radicular de los incisivos laterales.

Además se describen diferentes características relacionadas con la retención de caninos y se sugieren medidas preventivas para tratar de aminorar estas retenciones.

Palabras clave: Reabsorción radicular, caninos impactados.

\section{SUMMARY}

Upper canine impactation is a condition capable of promoting wide variety of clinical sequels ranging fromarch lengh discrepancy to adjacent teeth root resorption. This report presents two case in which both upper canines were clinically absent and resorbing lateral incisor root. In addition different characteristics relates to the condition of impacted canines are described and preventive measures for this condition are also suggested.

Key words: Root resorption, Canine impaction.

Fecha de recepción: Abril 2007.

Aceptado para publicación: Abril 2007.

* Dentista de Atención Primaria del Servicio Andaluz de Salud C. S. "La Paz". Distrito Bahía de Cádiz- La Janda. Cádiz.

** Doctora en Medicina y Cirugía. Máster en Salud Pública. Hospital Universitario "Puerta del Mar". Cádiz.

*** Diplomada Universitaria en Enfermería.

Rodríguez Romero FJ, Rodríguez Sánchez M, Rodríguez Rodríguez B. Reabsorción radicular de incisivos laterales superiores en relación con la erupción ectópica de caninos. Presentación de dos casos. Av. Odontoestomatol 2008; 24 (2): 147-156.

\section{INTRODUCCIÓN}

La impactación y erupción ectópica de caninos representa un gran problema por la ausencia de sínto- mas. Se conoce como diente impactado aquellos que se han formado dentro de hueso pero que han fracasado en el proceso de erupción (1). Cualquier pieza dentaria puede encontrarse impactada pero la 
mayor frecuencia de impactación de observa en terceros molares inferiores y superiores, seguidos por caninos superiores, segundo premolares superiores e inferiores y los incisivos centrales superiores $(2,52)$. Los caninos no erupcionados ocurren con una frecuencia 20 veces mayor en el maxilar superior que en la mandíbula y casi siempre están rotados de 60 a 90 grados sobre su eje longitudinal.

La incidencia de impactación de caninos superiores varia entre $0,92 \%$ a 2,2\%. (3,5-7). Con relación al sexo son dos veces más común en mujeres $(1,17 \%)$ que en varones $(0,51 \%)(4)$. La incidencia de impactación por palatino excede a la vestibular en una proporción de por lo menos 3:1 (8). El 8\% de todas las impactaciones caninas ocurren bilateralmente.

La etiología de la impactación canina es multifactorial y difícil de definir. Rara vez ocurre de forma aislada ya que es común observarla acompañada con agenesia o anomalías en forma, de incisivos laterales, con diente deciduos retenidos, con dientes supernumerarios o con una deficiencia esquelética premaxilar. Hay que destacar que los caninos superiores están predispuestos a quedarse incluidos por presentar el periodo mas largo de desarrollo, además de la vía de erupción más extensa y tortuosa $(9,10)$.

Diversos factores locales y generales están relacionados con la impactación de caninos superiores. Retención prolongada o perdida prematura del canino primario, discrepancia dentoalveolar, posición anormal del germen dental, patología localizada como quistes, neoplasias, odontomas y supernumerarios, agenesia o alteración en la forma de incisivos laterales, presencia de hendidura alveolar, anquilosis, trauma, origen idiopático, iatrogénico, aberración en la formación de la lamina dental, problemas naso respiratorios, variación en el tamaño de la raíz del diente, variación en el tiempo de formación radicular, secuencia de erupción anormal, exceso de espacio, cantidad de reabsorción del diente primario, forma de arco estrecha y herencia son factores locales reconocidos $(4,11-13,18,21)$.

Entre los factores generales se describen las deficiencias endocrinas (hipotiroidismo e hipopituitarismo), deficiencias vitamínicas (Vit D), factores genéticos, síndromes como disostosis cleidocraneal y craneosinostosis, presión muscular anormal, irradiación $(4,11-13,18,21)$.

Las secuelas relacionadas con la impactación de caninos son: malposición lingual o labial del diente retenido, migración del diente vecino y perdida de la longitud de arco, reabsorción interna, formación de quistes dentígenos, reabsorción radicular externa del canino retenido, reabsorción radicular externa de los dientes vecinos, infección relacionada con la erupción parcial de la pieza, dolor referido y combinación de las secuelas anteriores

Otra secuela importante es la reabsorción radicular por el tratamiento ortodóntico. La corrección ortodóntica de los caninos retenidos con un patrón de erupción ectópico, es un factor de riesgo para producir reabsorción apical de los dientes anteriores, la cual no fue solamente confirmada a los dientes laterales superiores adyacentes, la función del sistema de anclaje para ejercer fuerzas extrusivas dirigidas al canino retenidos, implican fuerzas intrusivas a los incisivos y de esta manera fuerzas de compresión al ligamento periodontal, estudios previos han asociado a las fuerzas de compresión con reabsorción radicular, ya que la reabsorción tiende a mantenerse activa todo el tiempo de tratamiento (21).

Las causas que provocan reabsorción radicular son: (21-26)

1. Traumatismos.

2. Inflamación crónica de la pulpa, tejidos periodontales o ambos

3. Presión inducida en el ligamento periodontal asociados a tratamiento ortodónticos. La alergia, la morfología de la raíz y el asma pueden ser factores de riesgo elevado, para el desarrollo de reabsorción radicular durante los movimientos de ortodoncia.

4. Tumores de crecimiento lento tales como, quistes, tumores de células gigantes, osteosclerosis y otras lesiones fibro-óseas.

5. Presión activa durante la erupción dental.

6. Dientes trasplantados.

7. Diente reimplantados.

8. Enfermedades sistémicas (hiperparatiroidismo).

9. Dientes impactados, dientes incluidos.

10. Discrepancia en la longitud de arcada. 
11. Hipoplasia maxilar.

12. Factores genéticos.

13. Dientes supernumerarios.

14. Factores inmunes.

Ericson S, estimó la retención canina en un 1\% (publicó la reabsorción de la raíz del incisivo lateral en el $12 \%$ de una muestra, que presentaban caninos impactados). Estimó que aproximadamente en el 0,7\% de los niños de 11-13 años, los incisivos permanentes han sido reabsorbidos, debido a la erupción ectópica de los caninos $(28,29)$.

La presión de los dientes permanentes en erupción ha sido considerada como uno de los factores más importantes en la reabsorción radicular (30). Hitoshi, en un estudio de 11 pacientes con reabsorción radicular de 12 incisivos centrales y 11 incisivos laterales, encontró que la reabsorción radicular fue mas frecuente en mujeres que en hombres en un radio de 10 a 1, siendo la edad promedio de esta reabsorción radicular a los 13,5 años con un rango de los 11 a los 23 años de edad. En algunos casos los dientes fueron extraídos debido a la extensa perdida radicular y reemplazados con los caninos retenidos los cuales fueron arrastrados ortodónticamente, en otros casos fueron restaurados protésicamente y en algunos casos no hubo tratamiento.

Sugiere que los dientes mal formados o con reducción en tamaño son más susceptibles a la reabsorción radicular, siendo los incisivos superiores e inferiores los mas afectados de toda la dentición. Radiográficamente los caninos superiores permanentes estaban parcialmente o totalmente con sus coronas en posición intima con las raíces de los incisivos afectados.

Los resultados de este estudio pueden indicar que los dientes permanentes son susceptibles a tener reabsorción radicular aun en ausencia de factores sistémicos cuando cierta presión es aplicada a sus raíces, ya que los dientes reabsorbidos nunca padecieron dolor, los pacientes no pudieron detectar esta situación hasta que la extensión del daño fue irreversible.

En orden de prevención de estos resultados, la detección temprana de estas retenciones de caninos es de vital importancia, de esta manera cuando existe falta de espacio para la erupción normal de los caninos permanentes, debido a la perdida prematura de los caninos deciduos o a una discrepancia entre el arco dental y los dientes, el examen radiográfico debería ser esencial para establecer un diagnostico y tratamiento adecuados, como son la extracción temprana de caninos o primeros premolares antes de que la reabsorción de los incisivos suceda (30).

Ilana Brin en un estudio con 20 pacientes, encontró 23 incisivos con reabsorción radicular, la edad promedio de estas reabsorciones fue a los 12,3 años con una desviación de los 11 a los 16 años de edad y sugiere que el fenómeno de reabsorción radicular de los incisivos laterales superiores, es un fenómeno que fue encontrado en el $12 \%$ de una muestra de pacientes con caninos retenidos, que el $40 \%$ de estos pacientes tenían una reabsorción en dirección oblicua y que esta reabsorción se manifiesta de una manera rápida y severa (31).

Ugalde FJ, publico un estudio de 601 pacientes con tratamiento ortodóntico donde encontró 35 pacientes con retención de caninos, 5,8\% de la muestra, a diferencia de la literatura que fue del 2,2 y 4,3\% (32). De estas retenciones el sexo fue 24 femenino y 11 masculino. Las retenciones fueron mayoritariamente unilaterales $77,1 \%$ y el canino derecho fue mas frecuente que el izquierdo un $42 \%$, la retención palatina fue en el $60 \%$ de los casos. El total de caninos retenidos fue de 43 piezas, el $20 \%$ causaron reabsorción radicular de los incisivos adyacentes, siendo los mas afectados el 11 en un 18,2\%, 12 en un 18,2\%, 2,1 en un $27.2 \%$ y 2,2 en un $36,4 \%$. Las exodoncias realizadas como consecuencia de la reabsorción tan extensa que presentaban fueron de 3 incisivos, 1.2 dos veces $(66,6 \%)$ y 2,2 una vez $(33,4 \%)(33)$.

Ericson en un estudio de 107 niños con una media de edad de 12,5 años, observo 156 caninos en posición ectópica y 56 en posición normal. El 93\% de los caninos en posición ectópica estaban en contacto con las raíces de los incisivos laterales adyacentes, y el $19 \%$ estaban en contacto con incisivos central. La reabsorción radicular sobre los incisivos laterales ocurrió en el $38 \%$ y el $9 \%$ en centrales. De los 58 incisivos laterales con reabsorción radicular esta reabsorción era leve en el $31 \%$ de los casos, moderada 
en el $9 \%$ y severa con afectación pulpar en el $60 \%$. Para incisivos centrales era de $36 \%$ leve, $21 \%$ moderada y 43 severa. De los 107 niños estudiados 51 (48\%) con caninos en posición ectópica presentaban reabsorción de los incisivos laterales maxilares. Existe correlación estadísticamente significativa entre la erupción ectópica de caninos, el contacto con los dientes y la reabsorción sobre los incisivos adyacentes (34).

\section{Medidas preventivas}

1. Radiográficamente:

- Técnica del Dr. Lindauer. Desde los 9 años de edad utilizando una radiografía panorámica consistente en observa la posición de canino no erupcionado, ya que si ha sobrepasado la línea media tangente de la raíz del incisivo lateral superior mesial de esta, las posibilidades de quedar retenido serán mayores (35).

- Uso de T.C. de los 9 a los 10 años de edad para detectar reabsorción de incisivos laterales (29).

- Técnica del Dr. Williams. Utilizando una rx posteroanterior de cráneo, de pueden prevenir retenciones de caninos desde los 8 años de edad, supervisando su trayecto (36).

2. Clínicamente:

- Observación: La ausencia de laterales puede causar retención dentaria debido a exceso de espacio, así como los laterales en forma de clavija, crecimiento excesivo en la base del hueso maxilar, erupción temprana de incisivos laterales o primeros premolares superiores, ya que los caninos vecinos pierden su guía de erupción y no encuentra su camino (37).

- Palpación: Generalmente el nódulo de un canino con un patrón de erupción correcto puede ser palpado a la edad de 9 años, para ver si se encuentra por bucal o palatino (38).

- Exodoncias: De los 10 a los 13 años de edad, se podría realizar la exodoncia del canino deciduo para regularizar el patrón de erupción del canino permanente (35). La exodoncia de canino permanente, en caso de que pudiera causar reabsorción de los incisivos adyacentes (30).

3. Análisis de dentición mixta adecuado, para efectuar un programa de extracción seriada en Clase
I apiñadas con enucleación de primeros premolares (36).

4. Remoción de una "barrera de tejido" como tejido fibroso gingival u óseo la cual impide la erupción del diente que parece que parece que va ha erupcionar y no lo hace, previa revisión del estado comparativo de la erupción del mismo diente en el segmento bucal contralateral para decidir si se interviene quirúrgicamente o no (39).

5. Autotrasplantación de canino; en caso de reabsorción severa de incisivos para evitar fuerzas intrusivas en estos dientes, y de esta manera evitar que se produzca una mayor reabsorción de las raíces de los dientes anteriores debido a las fuerzas mecánicas ortodónticas (40).

\section{Tratamiento}

Hay varias opciones abiertas en el tratamiento de caninos permanentes impactados, después de una evaluación de la oclusión. Las opciones son:

- Retiro de los caninos caducos (41).

- Ningún tratamiento, pero con evaluaciones periódicas por si aparecen cambios patológicos.

- Retiro quirúrgico y reemplazo prostético del canino impactado (4).

- Exposición quirúrgica del canino y su alineación ortodóntica.

- Autotrasplante del canino $(43,44)$.

Antes de tomar una decisión del tratamiento ha realizar, hay que tomar en consideración una serie de factores diagnósticos y radiográficos sobre el paciente:

- Edad.

- Salud general y bucal.

- Si existe espacio disponible o puede abrirse para la alineación del canino permanente.

- Posición radiográfica favorable del canino.

- Motivación del paciente a realizarse tratamiento ortodóntico.

- Contraindicaciones médicas para la cirugía.

Los factores, salud bucodental deficiente y la carencia de motivación, excluiría la alineación ortodóntica, aunque otros factores puedan ser favorables. Sin embargo aunque todos estos factores fueran favora- 
bles, la valoración radiográfica es muy importante para la planificación del tratamiento de estos pacientes $(28,42,45,46)$.

\section{Factores como:}

- Angulación del canino con respecto a la línea media.

- Altura vertical de la corona del canino.

- Posición antero-posterior del ápice de la raíz del canino.

- Posición de la corona del canino con respecto a los incisivos adyacentes.

- Reabsorción de la raíz de incisivos adyacentes.

- Posición vestíbulo palatal de la corona canina.

- Posición vestíbulo palatal del ápice del canino.

Puede influir en que el ortodoncista expone, alinee o quite un canino impactado superior permanente. (47)

\section{Diagnóstico}

Las imágenes tomográficas tomadas en 3D nos pueden mostrar lo siguiente: presencia o ausencia de caninos, tamaño del folículo, inclinación del eje largo del diente incluido, en este caso de los caninos, relación bucal y posición palatal, cantidad de hueso que cubre al diente, en que condiciones se encuentran los dientes adyacentes, consideraciones anatómicas (forma de la raíz) y desarrollo dental. $(48,49,50)$

\section{CASOS CLINICOS}

\section{CAso CLÍNICO 1}

Se trata de un paciente de sexo masculino de 11 años de edad, sin antecedentes personales de interés, acude a la consulta de Odontología en el Centro de Salud "La Paz" en Cádiz perteneciente al Distrito Cádiz-La Janda, refiriendo movilidad del incisivo lateral superior derecho (1.2) y que se le han desplazado hacia vestibular y los caninos no le salen. El paciente no refiere dolor, solo el desplazamiento del incisivo (Fig. 1).

Examen clínico: se observa una ligera vestibulización del incisivo lateral derecho (1.2) (Fig. 1), no hay cam-

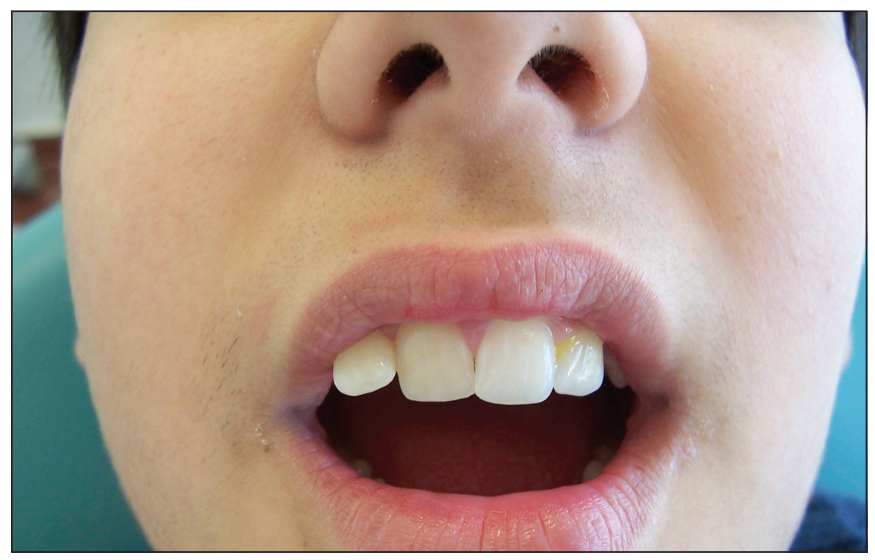

Fig. 1

bio de coloración de las coronas de los incisivos laterales. El canino deciduo derecho (5.3) permanece en arcada no así el izquierdo (6.3). Se observa la corona del canino derecho (1.3), haciendo prominencia por vestibular de maxilar superior (Fig. 2).

Se puede observa la erupción del canino permanente (1.3), una vez hecha la exodoncia del canino temporal, haciendo contacto con la corona del incisivo lateral permanente (1.2). Y se puede apreciar en la Fig. 3 que la corona del canino, provoca impactación en distal del lateral (1.2) y la reabsorción de la raíz de éste.

En la radiografía panorámica se observan estructuras óseas normales, 32 piezas dentales permanentes y 1 pieza dentaria temporal correspondiendo al 5.3,

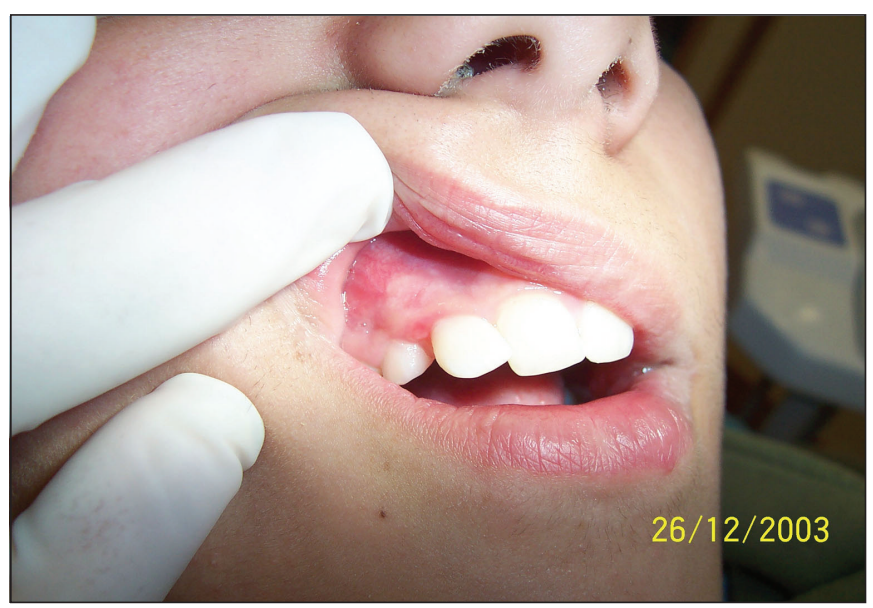

Fig. 2 


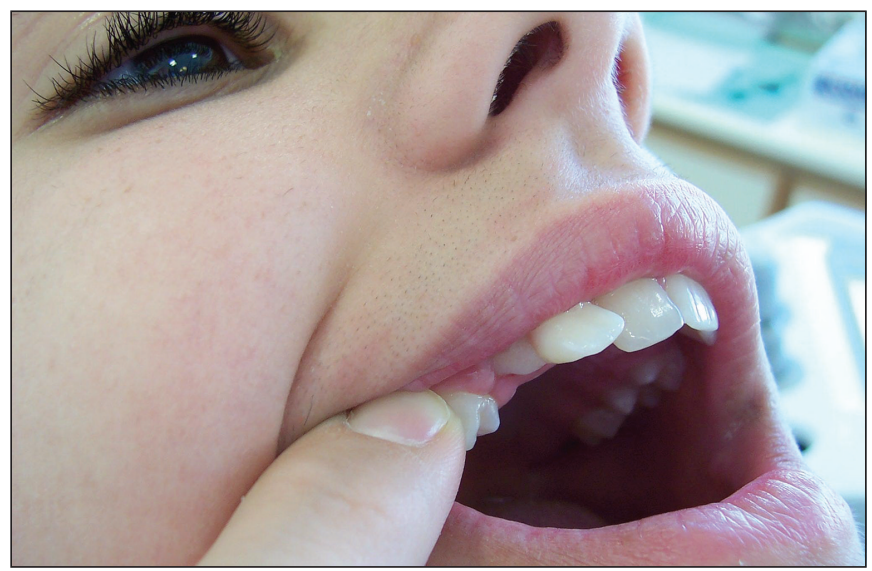

Fig. 3

erupción ectópica de las piezas dentarias 1.3 y 2.3 con formación completa de la raíz inclinación coronal mesial y reabsorción completa de la raíz del 1.2 y del 2.2, el paciente no presentaba caries (Fig. 4).

El tratamiento consistió en la exodoncia del canino temporal 5.3, extracción de ambos incisivos laterales 1.2 y 2.2 dada su severa reabsorción radicular y la imposibilidad de realizar tratamiento endodóntico de ambas piezas para su conservación, tracción ortodóntica de caninos permanentes en erupción ectópica, alineación en arcada de caninos permanentes con tratamiento ortodóntico, colocación provisional de prótesis removible que incluye las piezas 1.2 y 2.2, finalizando con la colocación de prótesis fija de ambos laterales (implantes osteointegrados) (Figs. 5, 6 y 7).

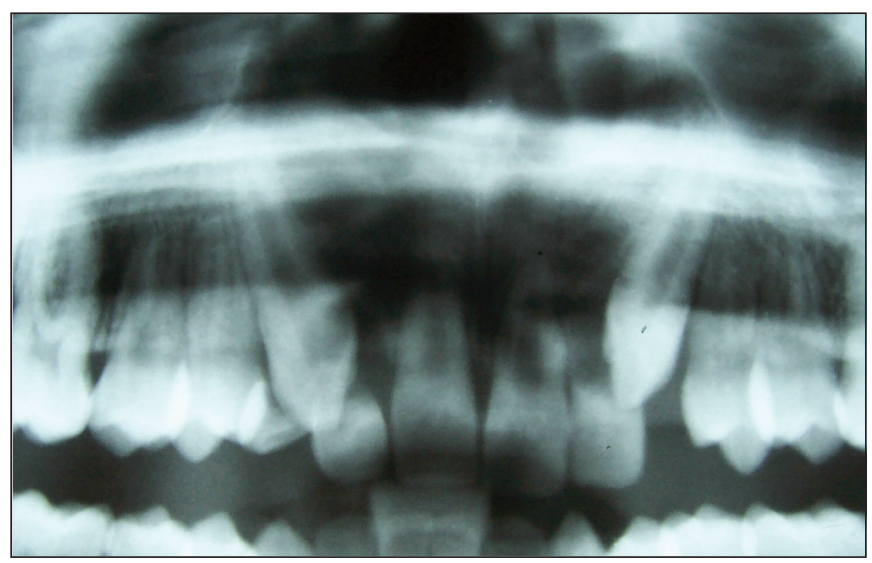

Fig. 4

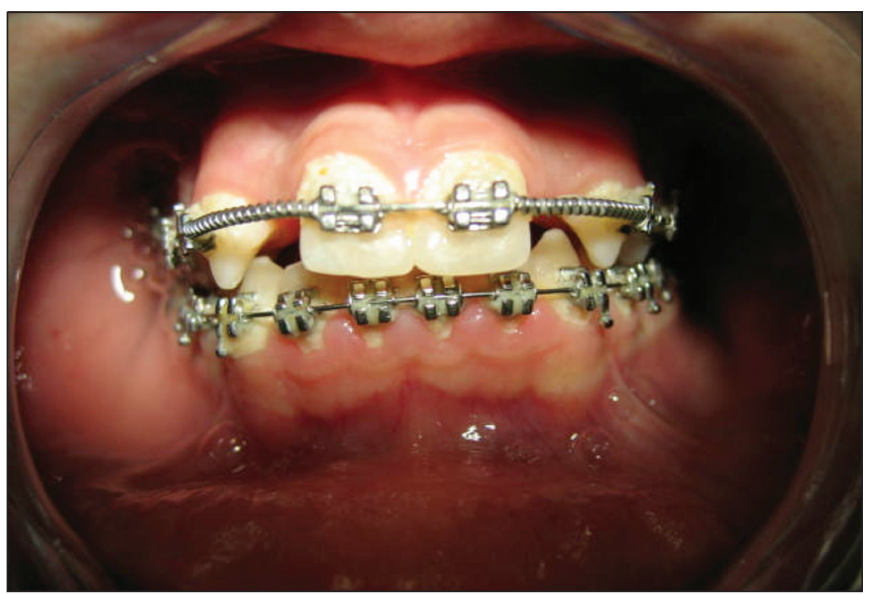

Fig. 5

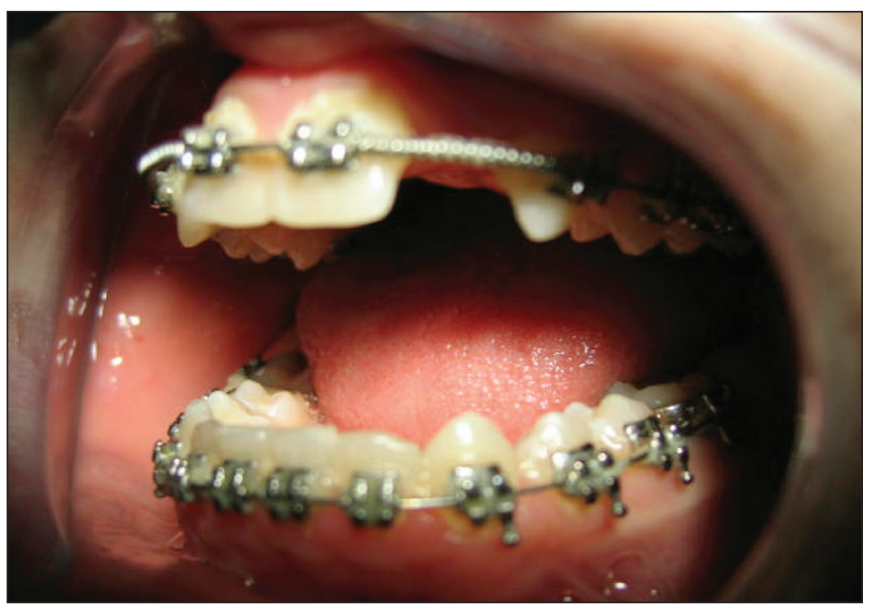

Fig. 6

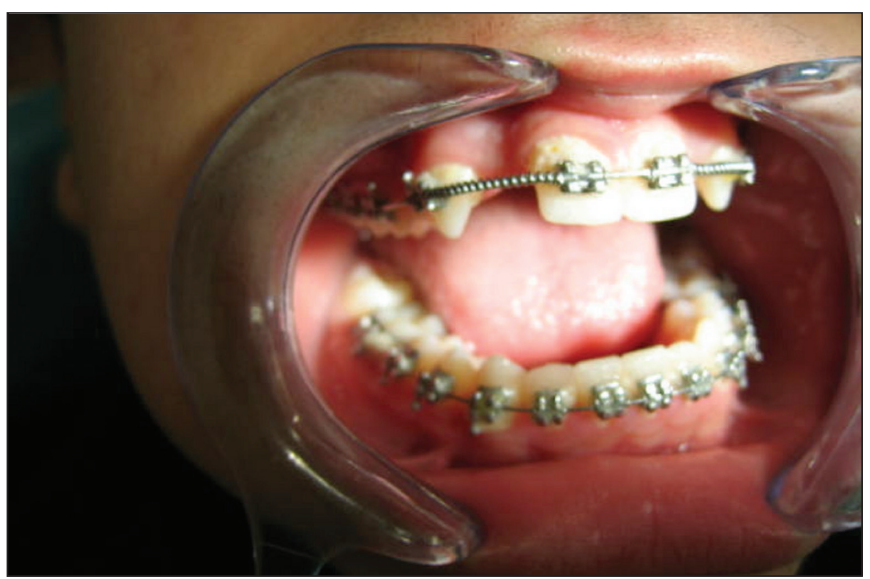

Fig. 7 


\section{CASO CLÍNICO 2}

Paciente de 11 años de edad, acude a consulta por proceso fistuloso en mucosa vestibular a nivel del incisivo lateral izquierdo (2.2), como consecuencia de la comunicación del saco folicular del canino superior izquierdo en erupción ectópica con la cavidad oral que le produce sintomatología local, el incisivo lateral no presenta cambios de color, no presenta movilidad, no presenta caries ni antecedentes traumáticos. En la Fig. 8 se aprecia una ligera vestibulización del incisivo lateral izquierdo.

Existe caries del 64 (clase II) con afectación pulpar. Se realiza ortopantomografía apreciándose imagen radiolúcida pericoronal a nivel del canino (2.3), en inclinación mesial, no se aprecia reabsorción radicular del 22, pero sí una mesio inclinación de su raíz por presión del folículo del 2.3 en erupción ectópica. También se aprecia reabsorción oblicua de tercio apical del incisivo lateral derecho (1.2) por erupción ectópica mesial del canino derecho (1.3) (Fig. 9). Se exodonciaron las piezas dentarias temporales 5.3 y 5.4 y dada la evidencia de falta de espacio en la arcada tanto superior como inferior y la reabsorción radicular de 1.2 el tratamiento consistió en endodoncia del incisivo lateral y tratamiento de ortodoncia (Figs. 8 y 9).

\section{CONCLUSIONES}

Ya que hoy en día no existe ningún método eficaz y seguro para diagnosticar la retención de caninos, se sugieren algunas medidas preventivas tanto radiológicas como clínicas para tratar de prevenir éstas en un estadio precoz y así tratar de evitar sus secuelas adversas:

1. El diagnóstico de impactación debe basarse en un examen clínico y radiológico. Se sugieren diversos signos clínicos como indicativos de impactación canina:

— Erupción retrasada del canino permanente.

- Retención prolongada del canino deciduo después de los 14 años de edad.

- Ausencia de prominencia canina.

- Imposibilidad de localizar la posición canina a través de palpación intraoral de los procesos alveolares.

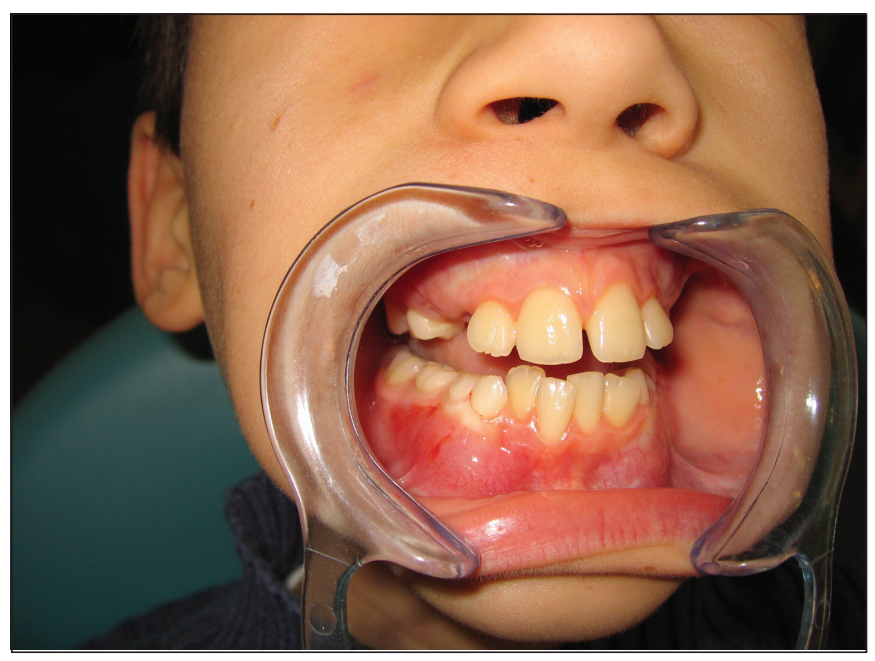

Fig. 8

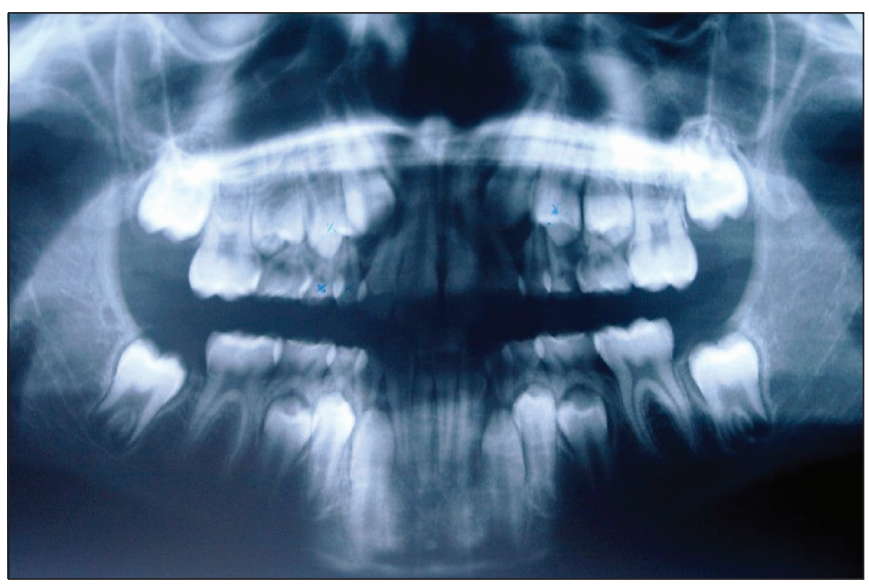

Fig. 9

—Presencia de una prominencia asimétrica.

- Presencia de una prominencia palatina e inclinación distal o migración del incisivo lateral.

2. La ausencia de prominencia canina a edad temprana no debe ser considerada como indicativo de impactación canina.

3. Con el uso de medios diagnósticos radiográficos (rx periapical, telerradiografía, ortopantomografía y tomografía computarizada), se pueden determinar la posición de los caninos y su relación con las estructuras anatómicas vecinas. 
4. La reabsorción de incisivos no se puede predecir, todos los caninos impactados tanto bucal como palatalmente deben ser considerados como potenciales.

5. La reabsorción externa de incisivo por erupción ectópica de caninos maxilares es rápida, silenciosa y asintomática.

6. Es necesaria la monitorización de la erupción de los caninos maxilares.

7. Las técnicas radiográficas y la exploración clínica son indispensables para un diagnostico precoz de esta patología y son unos de los requisitos previos básicos para el tratamiento endodóntico de la raíz afectada.

8. Se sugiere continuar investigando para encontrar métodos sencillos y fiables para él diagnostico de estas retenciones en el periodo de dentición mixta.

9. En el primer caso que presentamos, es impactante la reabsorción bilateral de incisivos tan acusada que presenta así como la edad y la ausencia total de sintomatología clínica. Él diagnostico tan tardío provoca estas consecuencias.

\section{BIBLIOGRAFÍA}

1. Sapp JP, Eversole LR, Wysocki GP. Patologia oral maxilofacial contemporánea España: Harcourt Brace, 1998:5.

2. Kuftinec M, Shapira S. The impacted maxillary canine: I Review of concepts. ASDC J Dent Chlid 1995;62(5):317-24.

3. Ballester JF, Toubia F, Irigoyen L. El canino incluido: diagnostico y tratamiento. $1^{\text {ra }}$ ed. Editora MDB. España 1990.

4. Bishara SE. Impactad maxillary canines: a review. Am J Orthod Dentofacial Orthop 1992;101(2): 159-71.

5. Bishara SE, Comer DD, McNeil MH, Montagano LN, Oesterle LJ, Yoongquist HW. Management of impacted canines. Am J. Orthod 1976;69 (4):371-87.

6. Rimes RJ, Mitchell CN, Willmot DR. Maxillary incisor root resorption in relation to thre ectopic canine: a review of 26 patient. Eur J Orthod 1997; 19(1):79-84.

7. Ericson S, Kurol J. Radiographic assessment of maxillary canine eruption in children with clinical signs of eruption disturbances. Eur $\mathrm{J}$ Orthod 1988; 8(3):133-40.

8. Fournier A, Turcotte JY, Bernard C. Orthodontic considerations in the treatment of maxillary impacted canines. Am J Orthod 1982;81(3):236-9.

9. Dewel BF, The upper cuspid: Its development and impaction. Angle Orthod 1949;19:79-90.

10. Jacoby $H$. The etiology of maxillary canine impactions. Am J Orthod 1983;84(2):125-32.

11. Becker A, Bimstein E, Shteyer A. Interdisciplinary treatment of multiple unerupted supernumerary teeth. Report of a case. Am J Orthod 1982;81(5): 417-22.

12. Fastlicht S. Treatment of impacted canines. Am J Orthod 1954;40:891-905.

13. Lewis PD. Preorthodontic surgery in the treatment of impacted canines. Am J Orthod 1971;60(4): 382-97.

14. Takayama T, Aiyama Y, Maxillary canine impactions as a possible microform of cleft lip and palate. Eur J Orthod 1982;4:275-7.

15. Becker A, Smith P, Behar R. The incidence of anomalous maxillary lateral incisor in relation to palatallydisplaced cuspid. Angle Orthod 1981; 51(1):24-9.

16. Langberg BJ, Peck S. Tooth-size reduction with occurrence of palatal displacement of canines. Angle Orthod 2000;70(2):126-8.

17. Brim I, Solomon Y, Zilberman Y. Trauma as a possible etiologic factor in maxillary canine 
impaction. Am J Orthod Dentofacial Orthop 1993;104(2):132-7.

18. Kaban LB, Cirugia bucal y maxilofacial en niños. México: Editorial Interamericana, McGrae-Hill 1992.

19. Becker A, Zilberman Y, Tsur B. Root length of lateral incisors adjacent to palatally-displaced maxillary cuspids. Angle Orthod 1984;54(3):28125.

20. Brin I, Becker M, Shalhav M. Position of the maxillary permanent canine in relation to anomalous or missing lateral incisors: a population study. Eur J Orthod 1986;8(1):1216.

21. Linge L. Patients charasteristics and treatment variables associated with apical root resorption during orthodontic treatment. Am J Orthod 1992; 35-43.

22. Cohen S. Odontologia General Mayo/Junio 2003 Vol. $\left.51 \mathrm{n}^{\circ} 3\right)$.

23. Ericson S, Bjerklin K, Falahat B. Does the canine dental follicie cause resorption of permanent incisor roots? A computed tomographic study of erupting maxillary canines. Angle Orthod 2002 Apr;72(2):95-104.

24. Harada K, Arita K, Nishino M. Severe root resorption of maxillary incisors and ectopic eruption of maxillary canine. Shoni Shikagaku Zasshi. 1989; 27(3):692-9.

25. Riyad A. Al-Qawasmi. Genetic predisposition to external apical root resorption Am J of Orthod and Dentof Orthoped vol 123,Issue 3, march 2003,Pages 242-52.

26. Riyad A. Al Qawasmi. Genetic predispositition to external apical root resorption: linkage of chomosome-18 market. J Dent Res. 2003 Mayo; 82(5):356-60.

27. Ericson S. Kurol JLongitudinal study and analysis of clinical supervisionof maxillary canine eruption.
Community Dent Oral Epidemiol 1986;14(3): 172-86.

28. Ericson S, Kurol PJ. Radiographic examination of ectopically erupting maxillary canines. Am J Orthod Dentof Orthop 1987;91(6):483-92.

29. Ericson S. Diagnosis of ectopally erupting maxillary canines-a case report. Europan $\mathrm{J}$ of Orthod 1988;115-21.

30. Hitoshi S, Root resorption of upper permanent incisor, caused by impacted canine. Am J Orthod 1984;299-306.

31. Brin I. Rebsorbed lateral incisor adjacent to impacted maxillary canines. Am J Orthod 1993: 60-6.

32. Mac Donald F. The surgical exposure and application of direct traction of unerupted teeth. Am J Orthod 1988:331-40.

33. Ugalde FJ, Gonzalez R. Prevalencia de retenciones de caninos en pacientes tratados en la clinica de ortodoncia UNITEC Investigación Vol. LVI, $n^{\circ}$ 2, Marzo-Abril 1999:49-58.

34. Ericson S, Kurol PJ. Resorption of incisors after ectopic eruption of maxillary canines: a CT study. Angle Orthod. 2000 Dec;70(6):415-23.

35. Lindauer S. Canine impactation identified early with panoramic radiographs. JADA 1992:93-7.

36. Williams B. Diagnosis and prevention of maxillary cuspid retention. The Angle Orthodontist 1981;30-9.

37. Henry J. Etiology of maxillary impacted canines. Am J Orthod 1983;125-39.

38. Rondeau B. Eruption of impacted maxillary cuspid. The functional orthodontist 1991;4-13.

39. Graber TM. Ortodoncia Teoria y Practica. $4^{\mathrm{a}}$ ed. Ed. Interamericana 1981;354-67.

40. Lownie JF. Autotransplantation of maxillary canine teeth. Int J Oral Max Surg 1986;282-7. 
41. Ericson S. and Kurol J. Early treatment of palatally erupting maxillary canines by extraction of primary canines. European Journal of Orthodontics. 1988;10:283-95.

42. Holmes A. Nashed R.R. Radiographic localisation of canines in general Dental Practice Dental Update 1990; February: 29-33.

43. Shaw B. Schneider S. S. And Zeyer J. Surgical management of ankylosed impacted mxyllary canines. Journal of American Dental Asssociation 1981;102:497-500.

44. Sagne S. Lennartsson B. Thilander B. Transalveolar transplantation od maxillary canines. Am J Orthod and Dentofacial Orthop 1986;90:14957.

45. Ericson S. Kurol J. Radiographic assessment of maxillary canine eruption in children with clinical signs of eruption disturbances. European Journal of Orthodontic 1986;8:133-40.

46. Jacobs S.G. The impacted maxillary canine. Further observation on aetiolpgy, radiographic localization, prevention/interception of impaction and when to suspect impaction. Australian Dental Journal 1996;41:310-6.

47. Stivaros N. Mandall N.A. Radiographic factors affecting the management of impacted upper permanent canines. British Journal of Orthodontics 2000;27(2):169-73.
48. Walker L, Enciso R, Mah J. Three-dimensional localization of maxillary canines with cone-beam computed tomography. Am J Orthod Dentofacial Orthop.2005 Oct;128(4):418-23.

49. Bayrak S, Dalci K, Sari S. Case report: Evaluation of supernumerary teeth with computerized tomography. Oral Surg Oral Med Oral Pathol Oral Radiol Endod. 2005 Oct;100(4):e65-9.

50. Sawamura T, Minowa K, Nakamura M. Impacted teeth in the maxilla: usefulness of 3D Dental-CT for preoperative evaluation. Eur J Radiol. 2003 Sep;47(3):221-6.

51. Nishioka M, Ioi H, Nakata S, Nakasima A, Counts $A$. Root resorption and immune system factors in the Japanese. Angle Orthod. 2006;76 (1):103-8.

52. Chun FC, Li TK, Lui VK, Newsome PR, Chow RL, Cheung LK. Prevalence of impacted teeth and associated pathologies-a radiographic of the Hong Kong Chinese population. Hong Kong Med J. 2003;9(3):158-63.

\section{CORRESPONDENCIA}

Francisco Javier Rodríguez Romero

General Muñoz Arenillas, 6, 9 Q.

11010 Cádiz

Tfno: 956-289519

Email:frrodrom@gmail.com 